\title{
The Biographical Network Method
}

\author{
by Neil Armitage
}

The University of British Columbia

Sociological Research Online, 21 (2), 16

$<$ ttp://www.socresonline.org.uk/21/2/16.html>

DOI: $10.5153 /$ sro.3827

Received: 29 Sep 2015 | Accepted: 31 May 2016 | Published: 31 May 2016

\begin{abstract}
This article introduces a network visualization method that enables a thorough analysis of the link between life history and social networks. Network visualizations are generally static, and as such they tend to disguise rather than uncover change and continuity within networks, and the influence that certain events may have on someone's sociability. The Biographical Network (BN) is a mixed method approach combining life story interviews with formal SNA that attempts to overcome the consequences of this lack of dynamism in network visualizations. In the first part of the article the underpinnings of the BN design and the logistics of the method are outlined in relation to a doctoral study on cultural cosmopolitanism. In the second part findings from applying the BN method with 28 young British and Spanish adults living in Madrid and Manchester are used to demonstrate its utility and its limitations for sociological analysis.
\end{abstract}

Keywords: Life History, Biography, Personal Networks, Qualitative Interview, Network Dynamics, Life Events

\section{Introduction}

1.1 Visual tools are ideal for producing, understanding and presenting data (Moody \& Healy, 2014), and for developing new perspectives on social phenomena. The purpose of this article is to present the Biographical Network (BN) Method, a visual method to explore, analyse and present the social history of networks. The method fuses biographical (life story) interviews and social network analysis (SNA) in order to explore and analyse social processes both at the individual and social level.

1.2 In the first part of the paper I outline and reflect on the design of the BN method, exemplifying the utility of visual metaphors and concepts for social research. I define a visual metaphor as a heuristic device that relates an idea to a specific visualization design, in this case, a participant - aided sociogram (Hogan et.al 2007). I then present the rationale for merging biographical with formal network methods to investigate social networks. In the second part of the paper, I outline a biographical network protocol for the purpose of sociological analysis and use findings from a study exploring the life histories and personal networks of 28 young British and Spanish adults to demonstrate the utility and limitations of the method.

\section{Visualising the Research and the Method}

2.1 I developed the method for a doctoral study exploring the lived experiences and consequences of 'cosmopolitan conviviality' upon the 'self' - that is the interaction with and subsequent maintenance of relations with 'others' that are objectively different in terms of nationality, language, ethnicity, religion, social class and so forth. In network analysis this may be referred to as 'differential association'. While Hannerz (1996) coined the 'cosmopolitan stance' as the willingness and openness to engage with 'others', the doctoral study aimed to uncover and fully unpack how such a stance evolves (or not) through a person's life history. The method draws heavily on the theoretical perspective of symbolic interactionism, whereby the network of 'significant others' close relations of family, friends, partners together with a chorus of 'less significant others' could be seen as a 'looking glass' on the 'self' (Cooley 1983). The method was designed specifically to explore the evolution of 'self' towards (or not) a cosmopolitan stance. 


\section{Visualising Evolution - From Metaphor to Model}

To solve this puzzle I began by seeing life as a pebble falling into water, which on entry causes a series of concentric circles that represent the chapters in life through which the 'self' evolves. The pebble's entry in to the water represents birth that automatically displaces the lives of the primary care providers who form an immediate deep protective circle. It is within these initial deeper circles that primary socialisation occurs (Cooley 1983). As life progresses, every consequent circle becomes shallower and wider, reflecting an increasing independence from significant others and the broadening of horizons for social action and connection. This outward direction represents the natural life-path from home out in to the world (Tuan 1996), as actions and actors in previous chapters influence the contexts and actions of the following chapters. The ripples between each may coincide with formal transitions such as moving from primary to secondary schooling, to college and in to employment, or reflect other events such as relationship formation and breakdown, migration, illness, war, parenthood, etc. It is during such transitions the influence and meaning of significant others are reconfigured, and the life history as a whole. However, as time progresses, the ripples fade, and with it life and the relations that constitute it.

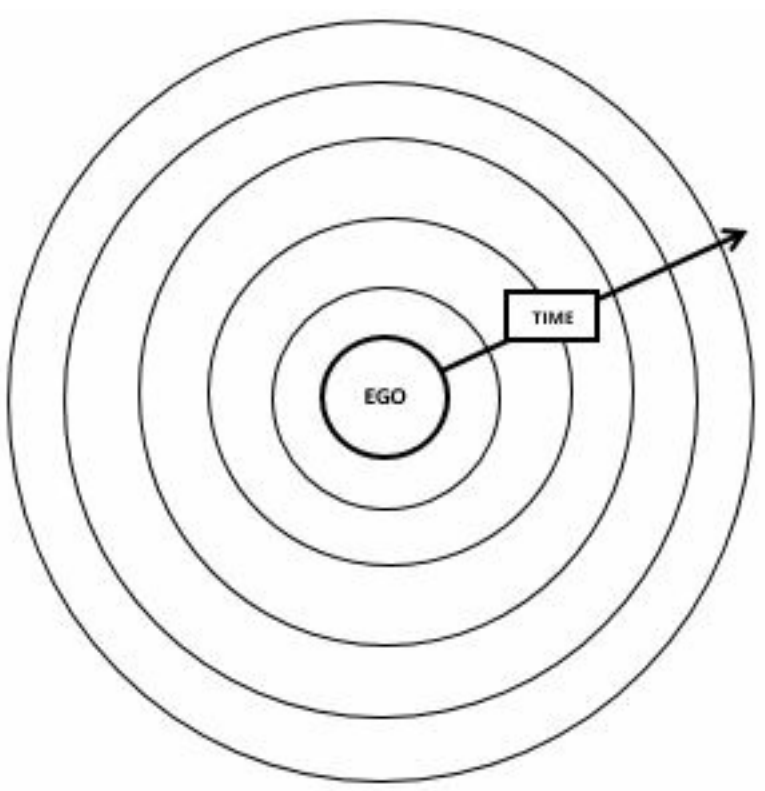

Figure 1. The Visual Tool

2.3 This visual metaphor of life reflects the descriptive and interpretative principles of sociological analysis, that is, to explore and understand behaviour and attitudes in relation to its social and historical contexts. However, like any metaphor, it emphasises and focuses certain avenues of description and interpretation other overs, such as the notion of linearity in the evolution of 'self'. Here it was used primarily as a heuristic device, a model to work with and expand on for the purposes of the research project and the questions thereof.

2.4 The metaphor come model provided a framework to explore the evolution of 'self' as a series of life chapters, and initiated the foundation of a visualisation method to organise and construct personal networks (egonets) using a biographical framework. This was done with the intention of analysing fully the link between socialization and sociability (Bidart and Charbonneau 2011) -or cosmopolitan conviviality and the consequences thereof. I now turn to the complementarity of biographical methods and social network analysis, both in its formal quantitative and less formalised qualitative guises.

\section{A Rationale for mixing Biographical Methods and Social Network Analysis}

Biographical methods, particularly life story interviews are ideal to explore the numerous contexts that people engage in through life; family, education, leisure, work etc., and the ways these different arenas intersect in peoples' everyday lives (Henderson et al. 2007). Yet life story narratives are constructed accounts; presentations of self that highlight as much the circumstances and stances through which life events are articulated as the events themselves (Brannen \& Nilsen 2011). While this makes life story interviews particularly useful for exploring reflexivity and socialisation, used alone, it is difficult to expose the provisional nature of the narrative. This ultimately limits what one can see (Holland and Thompson 2009:464). Therefore methods which can contextualise the life story, and enable an exploration of the provisionality of the narrative enriches the social analysis. Here visual methods such as time-lines (Bagnoli 2009, Brannen \& Nilsen 2011) and relational mapping 
The tangibility of the map is an important feature. This allows the interviewer and participant to return to features of the map in the interview and subsequent interviews to unfold the process of description-elaboration-theorisation (Emmel 2008)

While biographical studies often recognise the importance of significant others and social relations more broadly in the shaping of life histories, seldom are a person's ties formally analysed as a contextual layer through which to cross-reference the life story. Here social network analysis (SNA) in its formal quantitative guise can assist with both data construction and analysis. Taking the theoretical premise that 'self' is a social and relational construct, arising and maintained through interaction with others (Mead 1984), formal SNA provides a set of tools to measure and analyse the network of social relations in which people are embedded. The basic premise of the network approach is that actors, be they individuals or organisations, are interdependent with other actors and that this interdependency has consequences for social behaviour and attitudes, and hence social change (Boissevain 1979, Laumann 1973).

Both network visualisations and analysis provide presentation of 'self' that can be analysed both quantitatively and qualitatively. If analysed in tandem with the life histories, a means to cross-reference and 'capture contradiction, dissonance and repetition' (Holland \& Thomson 2009:464) between data sets is enabled, which in turn improves the internal and external validity of the research (Ryan et al. 2014). Further, as argued throughout this special edition, used alone, formal SNA struggles to capture the relational content and histories that form the current composition and structure of the network, and imbue it with meaning (Crossley 2010, Edwards 2010).

Networks of personal relations evolve over time. They reflect and go with socialisation. Their history and dynamics contribute to their present structure. (Bidart \& Lavenu $2005: 359$ )

2.8 As previously noted the biographical network approach sees personal networks as a 'looking glass' (Cooley 1983) or a lens to view both the temporal and social structure of 'self'. I now provide greater detail on how the methods were qualitatively mixed (Mason 2006) for sociological analysis.

\section{From Rationale to Practice - Merging the Methods for Sociological Analysis}

2.9 The benefits of using and mixing qualitative methods with formal SNA are now well established within the paradigm of social networks (Bellotti 2014, Dominguez \& Hollstein 2014, Edwards 2010). This special edition illustrating numerous approaches that have been taken. While the biographical network method contributes to this field, it should be noted, that it was designed specifically to contextualise and explore the evolution of 'self' and not networks per se. That noted, the method and the protocol could be adapted to explore the evolution of an organisation or a social movement, and/or focus more specifically on the dynamics and evolution of networks.

2.10 Through piloting the visualization method I realised that the life story interview and the network visualization needed to be independent data sets if the purposes of the study were to be fulfilled. Piloting the visual tool I first asked participants to self-identify significant life chapters and significant others. The number of chapters creating the series of concentric circles in which currently significant alter were to be distributed according to when they first became significant in the person's life. It should be iterated, as it is stands, the method neither intended to nor enables an exploration of networks at different life stages. Instead, it explores and uncovers more fully how the current network, its composition and structure, originated and evolved to its current state.

2.11 Its main purpose was to explore how the biographical distribution of relations and their longevity within an individual's biography corresponds or not with their life path and the story thereof. For example, is a transformative event such as attending university matched by a large influx and consolidation of relations within the network, and/or any change in sociability (composition and structure) in later life chapters? And if so, how/why? The biographical structure allowed a thorough interrogation of the link between socialization and sociability during the interview.

2.12 Piloting demonstrated that by starting with the visualization, the following life history narrative drew heavily on the completed network visualization. The life history was thus not independent of the network, and the opportunity to uncover contradiction, dissonance and repetition between the life history and network was severely diminished. In sum, when starting with the visualization, a life history of the network and not the person 
tended to be produced. While insightful in understanding the dynamics and evolution of the network, with the visualization proving a very useful visual tool to construct and elicit narratives (see Tubaro et al. and Altissimo this issue), a significant part of the contextual puzzle through which to interpret the network remained absent.

2.13 For the purposes of the doctoral study the life history interview preceded the network visualization to maintain each methods independence for cross-referencing. By taking this order the network could be interpreted in relation to the broader context from which it is abstracted (Edwards 2010). With two separate presentations of 'self', the dynamics and evolution of the network could be more fully interrogated for sociological analysis in the final stage of the interview.

\section{The Biographical Network Method in Practice}

3.1 For purposes of clarity I illustrate how the method worked in practice, from producing, to exploring and then presenting the data. In the concluding discussion the method's benefits and limitations, both for network studies and sociological analysis, are summarised.

\section{Producing the Data}

\section{The Sample \& Study Locations}

3.2 The method was used with 28 young British and Spanish adults (15 men and 13 women) between the ages of 23 and 35 residing in Madrid or Manchester. Young adults were chosen as entry into working and adult life is typically characterised by a significant renewal of personal relationships, as work colleagues and interest based friends complement and replace relationships from adolescence and education (Degenne \& Lebeaux 2005, Grossetti 2005, Bidart \& Lavenu 2005). This makes young adults particularly interesting in terms of exploring how sociability and 'self' evolve.

3.3 The sample was split between 'non-movers', people who reside and work in their native context, and 'movers', people who reside and work in a different national context to the one of their childhood and adolescence. All of the interviewees (14 movers and 14 non-movers) had resided a minimum of one year in Madrid or Manchester, ensuring they all had opportunities to experience and reflect on changes in their networks and relationships since living in either city.

3.4 Both Madrid and Manchester attract and have a significant population of young foreign adults from Europe and beyond for study and work. With diverse populations in terms of nationality, languages spoken, ethnicity, religion and so forth, they provided ideal locations to explore young adults' openness's and willingness to engage in 'cosmopolitan conviviality'.

\section{The Questionnaire and Interview Preparation}

3.5 Prior to interview participants were informed of the study via an email titled; 'The Cross-Cultural Interaction of young British and Spanish adults living in Madrid and Manchester' and asked to complete the attached questionnaire. The survey included demographic details (age, occupation, education, languages spoken, etc.) a network name-generator and an autobiographical prompt. The questionnaire was used to elicit data that would be used to semi-structure the life-history interview and the network visualization. This meant that the interview and network visualization materials could be prepared prior to the interview, allowing more time in the interview to be dedicated to the life history and analysis and discussion of the network visualization.

3.6 A multiple name generator tool consisting of six relational questions (Q1-Q6) was designed specifically for the research question - drawing on the Social Support Interview Scale (ASSIS) by Barrera (1980) and a multiple name generator developed by Marin and Hampton (2007).

- Q1. With whom do you discuss important personal matters?

- Q2. With whom do you enjoy socialising and travelling?

- Q3. Name friends/colleagues/family with a different nationality to you

- Q4. Name friends/colleagues/family with a different social class background to you

- Q5. Name friends/colleagues/family whom you consider different to you in some way

- Q6. Name anyone who is or has been especially close to you

3.7 Interviewees could name as many people as they wished under each question, and apart from Q1 and 
Q2, names could be repeated on Q3 to Q5. This was done to explore differentiation between confidants (Q1) and companions (Q2), and explore the extent to which foreign nationals (Q3), different social classes (Q4) and people they subjectively considered different (Q5) were also named as confidants (Q1) and companions (Q2). The more settled participants in the sample like Iwan below, a non-mover in his native Manchester, found it harder to distinguish between confidants and companions as these groups were often symbiotic.

\footnotetext{
These first two lists, I found that interesting because I thought they are a very similar group of people, people who I'd give my time to, especially like travel and socialise with which is important time isn't it, are the kind of people who I'd also talk to about what's going on in my life so it could be a little while to think of other people who perhaps, who perhaps weren't, I don't know what you call it, top level people who I would socialise with (Iwan 34)
}

Whereas less settled and more socially active participants like Gasper below, a Spanish mover to Manchester, found it easier to distinguish between their purely 'going out' friends (companions) from their 'true' friends (confidants), both those present in the city and located elsewhere.

It is not difficult to meet people, sometimes it's difficult to keep the people, because you can meet for one day, two days but maybe after three weeks it's gone the possible relation, the friendship, it's gone /.../ it's normally quite nice, quite easy to meet people, and spend the night out with different people and have a good time, but maybe next day it's different... (Gasper 30)

3.9 While the instruction to not repeat names under Q1 and Q2 generated considerable reflection and discussion in the interview, arguably greater internal validity and reliability would have been achieved by allowing repetition. To allow interviewees to name people who were or had been significant in their life, but who were not captured through the first five prompts, the final catch-all prompt (Q6) was used.

3.10 The final part of the questionnaire asked, 'If you were to write your autobiography what would be the titles of the book's chapters?'. If they so wished, participants could provide a title of the autobiography. Again interviewees could name as many chapters as they liked. These chapters would semi-structure the life-history interview, and form the concentric circles for the network visualization. The returned questionnaire data was then used to prepare the interview material. Additionally, the questionnaire encouraged interviewees to reflect on their networks and life history in preparation for the scheduled face-to-face interview. A limitation of the survey tool is that it did not mirror the interview protocol, with the name generator preceding and potentially biasing the naming of the chapters. However, while some chapter titles were directly related to significant others; the meeting and ending of intimate relationships, the divorce or death of parents, etc. many times these significant others were not named via the name generator.

3.11 The names elicited from the name generator were transferred to different coloured 'memo tip' notes representing nationality, class, other difference etc. This enabled alter attributes to be visualized on the network visualization. Starting with ego, a series of concentric circles representing each chapter were drawn on a large A2 size paper, with a few extra circles added to the number of chapters provided to allow for manoeuvrability during the interview (see Figure 1). The interview preparation, like the questionnaire, took around 15 -30 minutes to complete.

\section{The Life History Interview}

3.12 The interview started with the life history, followed by the visualization of the network, and a discussion linking the life history and the network. The average interview was 2 hours and 9 minutes, with the shortest taking just over an hour due to time restrictions and the longest was over 3 hours. They were conducted in English or Spanish and digitally recorded.

3.13 After introductions and outlining the interview schedule, interviewees were prompted to use their autobiographical chapter titles to narrate their life history. All but one of the life histories were organised and narrated chronologically. Interviewees were informed that while narrating their chapter titles, that if they mentioned people not originally provided via the name generator, then I would write them down and they could be added to the network visualization later.

3.14 The chapter titles provided a structure to narrate the life-story. If the interviewee needed encouragement, or was confused by the instructions, clarification was provided using the following prompt; 'I see your chapter titles as the skeleton of your life, I would like you to now put some flesh on the bones'. Interviewees provided between four and fifteen chapter titles, with an average of eight across the sample. With the number of chapter titles known, the first part of the interview schedule could be organised and controlled with appropriate prompting. 
The aim was to evolve a relatively informal interview style, or a 'conversation with a purpose' (Burgess 1984:102 as cited in Mason 2002: 62), and this was achieved across the sample.

\section{The Network Visualization}

3.15 After completing the life history, a short break was taken before I introduced and described the network template and the named coloured 'memo tip' notes. Prior to starting the visualization, interviewees were asked which of the extra alter mentioned during the life history they would like to add to their network. These were written on a different coloured note from those already prepared. Chapter titles were then either written directly onto the template, or if the life history had brought up other significant events in their life path, interviewees were given the chance to add, merge, or subtract chapters on the paper template.

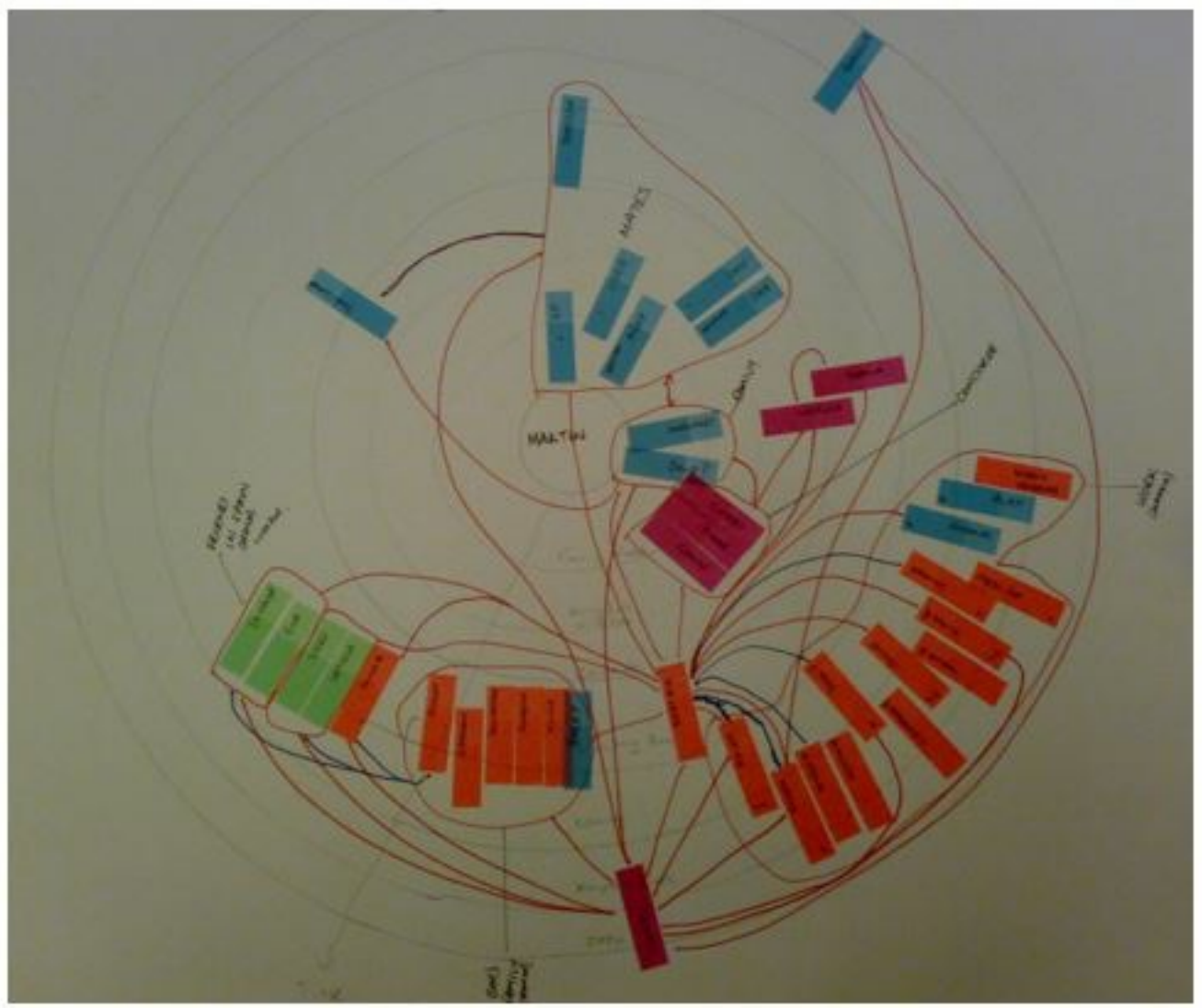

Figure 2. Photo of a biographical network visualization

3.16 Thereafter, the named memo-tips (the network nodes) were placed according to the chapter in which the person first entered and became significant in their life. Thus to reiterate the network is biographically structured, such that all those named either remain currently active and/or are considered significant within their life path and history.

3.17 Interviewees were also prompted to position alter who knew each other on or around the same radius. The context of where and how ego knew alter was attained as names were placed on the template, unless already clear from the life history. When all the names had been placed, the specific attributes of alter provided for Questions 3 to 5 were established. Abbreviations for nationalities and social class background, language of interaction, current location were written directly on the notes. Through getting each person to explain why they considered certain alter to be of a different social class background, or considered different in some way, subjective constructions of difference were explored. Thereafter they were asked to identify any other objective differences which they may or may not have already identified within their sociogram, such as ethnicity, age, religion, sexuality, etc.

3.18 With all the memo notes arranged accordingly, including those added from the life history, ties between alter and groups were then elicited. Following the participant-aided sociogram protocol developed by Hogan et al. (2007), a distinction was made between strong and weak ties and groups using a red and blue marker pen.

Strong and weak groups were first established and given names. Thereafter strong (red) or weak (blue) ties were drawn between groups, groups and alter, and between alter and alter. The relational matrix constructed from this 
data enabled formal structural analysis in UCINET and qualitative analysis of the networks in NetDraw (Borgatti et al. 2002).

\section{Discussion and Analysis}

4.1 When all the ties had been established to the satisfaction of the interviewee, they were asked for their reflections on the network, becoming qualitative co-analysts. They were allowed time to make observations and comment on their network. Similarly this allowed for me to prompt them on certain aspects of the network that emerged through its visualisation, and cross reference these with aspects of their life history previously narrated and fresh in memory. This allowed for a more thorough exploration into how their cosmopolitan conviviality related to certain biographical chapters, life-events and social contexts, and the effects of this on their sociability.

4.2 Towards the completion of the interview interviewees were asked to identify the most important people within their network. This information verified the validity and reliability of the name generator tool used, with interviewees generally naming confidants (Q1) and those who were or had been especially those (Q6). These reflections prompted a discussion that led into the concluding moments of the interview. Leaving the digital recorder running interviewees were asked how they found the interview and if they had any questions regarding the research. Their concluding remarks proved fruitful as they reflected on the topics covered and the interview process itself, embodying the rapport that had developed during the course of the interview. A digital photograph of each network visualization was also taken for data management purposes.

\section{Exploring the Data}

4.3 With a wealth of qualitative and quantitative data produced, social network analysis (SNA) provided a formal and structured means to compare the 28 personal network (Bellotti 2014 and this issue). I first outline the analysis of the networks and how the biographical structuring of the network visualization and the life history narratives enriched this process.

\section{Comparative Social Network Analysis}

4.4 The composition of the networks were first analysed and compared using standard SNA measurements to explore the extent to which people engaged with 'others'. Network homophily/heterophily in terms of nationality, social class, language of interaction, ethnicity, etc. were calculated using the E-I index[1] (Krackhardt \& Stern 1988) and cultural heterogeneity was measured using Blau's index ${ }^{2]}$. Both measurements enable networks of variable sizes to be compared. With alter named in Q1 and Q2 often repeated under Q3 - Q5, alter were used in numerous compositional calculations. However, in terms of the network size, they were only included once. The biographical networks ranged in size from 21 alter to 60 alter, including alter added from the life history, with a mean of 36.9 alter across the sample. $(21 \leq B n \leq 60, x=36.9)$.

However, initial analysis of biographical networks (Bns) highlighted that both homophily and heterogeneity measures were skewed by the extent to which people named family members or not, i.e. alter of a similar nationality, etc. While family relations (parents, siblings, cousins, and so on) were significant in terms of the life history and the network structure, to make substantive compositional comparisons in terms of nationality (Q3) and social class (Q4), they were omitted to produce the convivial network (Cn) $(17 \leq C n \leq 53, x=31.8)$. Transnational family members, and family acquired through partners and spouses were included in the analysis.

For the purposes of the research and analyse the extent that cosmopolitan conviviality led to 'others' becoming significant 'others', the friendship network (Fn) was constructed ( $4 \leq F n \leq 44, x=18.14)$. This comprised of confidants (Q1), companions (Q2) and especially close relations (Q6). The three nested network sizes used in the comparative analysis were;

- The Biographical Network $(B n)=(Q 1-Q 6)+($ alter derived from the life history)

- The Convivial Network $(\mathrm{Cn})=\mathrm{Bn}-$ family relations

- The Friendship Network $(F n)=(Q 1+Q 2+Q 6)$-family relations

\section{Biographical Network Analysis (BNA)}

4.7 Through tabulating the compositional values of the convivial and friendship networks, cases began to cluster along the degree of homophily/heterophily and cultural heterogeneity within the convivial $(\mathrm{Cn})$ and friendship (Fn) networks. In terms of nationality and language, three qualitatively deduced groups emerged; faint, 
stable or wide convivial horizons (see Tables 1 to 3 ). Through cross-referencing network data and the life story narratives, the emergence of 'other nationals' within the networks could be located in both time and space.

Further, stories behind how some 'other nationals' had become companions and confidants, or had diminished in importance were elicited. For example, it was shown how geographical mobility both within and across national borders, both their own and that of significant others generated different degrees of co-presence and co-absence with 'co-nationals' and 'other nationals'. The iterative process of circulating between network measurements across the three network sizes ( $\mathrm{Bn}, \mathrm{Cn} \& \mathrm{Fn}$ ) and the narratives enabled these groups to be more clearly delineated and defined.

For example, whereas faint horizons named 'other nationals' in their convivial network, either friends originating from foreign studies or current work colleagues, unlike cases with stable and wide horizons, their friendship networks remained homipholous (Fn National E-I $\leq-0.78$ ). This was either due to less opportunities to engage with 'other nationals' generally and/or preferring to confide in and socialise with old 'co-national' school and university friends.

Table 1. Network Composition Measures of Faint Horizon Cases

\begin{tabular}{|c|c|c|c|c|c|c|c|c|c|c|}
\hline \multirow[t]{2}{*}{ CASE } & \multicolumn{4}{|c|}{ Convivial Network ( $\left.C_{n}\right)$} & \multicolumn{4}{|c|}{ Friendship Network (Fn) } & \multicolumn{2}{|c|}{ Heterogeneity } \\
\hline & Size & $\begin{array}{l}\text { Other } \\
\text { Nationals }\end{array}$ & $\begin{array}{c}\text { National } \\
\text { E-I index }\end{array}$ & $\begin{array}{l}\text { Ianguage } \\
\text { E-I index } \\
\text { (b) }\end{array}$ & Size & $\begin{array}{l}\text { Other } \\
\text { Nationals }\end{array}$ & $\begin{array}{l}\text { National } \\
\text { E-I imdex } \\
\text { (c) }\end{array}$ & $\begin{array}{l}\text { Languzge } \\
\text { E-I imdex } \\
\text { (d) }\end{array}$ & $\begin{array}{l}C_{n} \\
(e)\end{array}$ & $\begin{array}{l}F n \\
(A)\end{array}$ \\
\hline Robow & 43 & 17 & -0.21 & -1 & 18 & 2 & -0.78 & -1 & 0.558 & 0.198 \\
\hline Hensal & 26 & 8 & -0.38 & -0.58 & 10 & 1 & -0.80 & -0.80 & 0.426 & 0.130 \\
\hline Inar & 31 & 8 & -0.48 & -1 & 15 & 1 & -0.87 & -1 & 0.408 & 0.125 \\
\hline Dybaw & 32 & 16 & 0 & -1 & 4 & 0 & -1 & -1 & 0.650 & 0 \\
\hline Mogele & 30 & 6 & -0.60 & -1 & 18 & 0 & -1 & -1 & 0.340 & 0 \\
\hline Alisis & 23 & 4 & -0.83 & -0.91 & 11 & 0 & -1 & -1 & 0.287 & 0 \\
\hline dean & 31 & 9.8 & -0.42 & -0.38 & 12.7 & 0.7 & -0.91 & -0.97 & 0.445 & 0.084 \\
\hline
\end{tabular}

4.9 Whereas the difference between cases with wide horizons from those with stable horizons was the greater degree to which 'other nationals' were also embedded in their friendship networks (Fn National E-I $\geq$ 0.03), together with the greater cultural heterogeneity of these networks (mean of 0.626 ) - positively correlated to the heterogeneity of their convivial networks $(0.464 \leq \mathrm{e} \leq 0.768)$. Yet as opposed to those with stable horizons, many of these relations were more recently added within the biography and arguably their networks were less stable. The ability to communicate in another language was influential in the degree of cultural heterogeneity, with settlement and improving second language comprehension particularly decisive for the British living in Madrid.

Table 2. Network Composition Measures of Stable Horizon Cases

\begin{tabular}{|c|c|c|c|c|c|c|c|c|c|c|}
\hline \multirow[t]{2}{*}{ CASE } & \multicolumn{4}{|c|}{ Convivial Network ( $\mathrm{Cm}$ ) } & \multicolumn{4}{|c|}{ Friendship Network ( $F k$ ) } & \multicolumn{2}{|c|}{ Heterogeneity } \\
\hline & Size & $\begin{array}{c}\text { Other } \\
\text { Nationals }\end{array}$ & $\begin{array}{c}\text { Nations! } \\
\text { E-I index } \\
\text { (a) }\end{array}$ & $\begin{array}{l}\text { Ingouge } \\
\text { E-I index } \\
\text { (b) }\end{array}$ & Size & $\begin{array}{c}\text { Otber } \\
\text { Nationals }\end{array}$ & $\begin{array}{l}\text { Nations! } \\
\text { E-I index } \\
\text { (c) }\end{array}$ & $\begin{array}{l}\text { Languzge } \\
\text { E-I index } \\
\text { (d) }\end{array}$ & $\begin{array}{l}C s \\
\text { (e) }\end{array}$ & $\begin{array}{l}F n \\
\text { (f) }\end{array}$ \\
\hline Riz & 18 & 8 & -0.11 & -0.11 & 10 & 4 & -0.20 & -0.20 & 0.599 & 0.540 \\
\hline Mlaties & 32 & 9 & -0.44 & -0.50 & 28 & 9 & -0.36 & -0.43 & 0.419 & 0.456 \\
\hline Launa & 30 & 6 & -0.60 & -0.67 & 14 & 4 & -0.43 & -0.57 & 0.320 & 0.408 \\
\hline Lux & 25 & 13 & 0.04 & -0.36 & 11 & 3 & -0.45 & -0.45 & 0.499 & 0.397 \\
\hline Robert & 51 & 16 & -0.37 & -0.50 & 44 & 12 & -0.45 & -0.84 & 0.478 & 0.435 \\
\hline 2 laxk & 41 & 25 & 0.22 & -0.12 & 15 & 4 & -0.47 & -0.73 & 0.505 & 0.237 \\
\hline Enat & 41 & 10 & -0.51 & -0.85 & 37 & 10 & -0.46 & -0.84 & 0.386 & 0.409 \\
\hline Claire & 30 & 7 & -0.53 & -0.93 & 24 & 6 & -0.50 & -0.92 & 0.571 & 0.393 \\
\hline Gaspex & 32 & 14 & -0.13 & -0.13 & 18 & 4 & -0.56 & -0.56 & 0.490 & 0.346 \\
\hline Ret: & 30 & 12 & -0.20 & -1 & 10 & 2 & -0.60 & -1 & 0.545 & 0.320 \\
\hline Mlean & 33 & 12 & -0.26 & -0.55 & 21.1 & 5.8 & -0.45 & -0.65 & 0.462 & 0.409 \\
\hline
\end{tabular}

Table 3. Network Composition Measures of Wide Horizon Cases 


\begin{tabular}{|c|c|c|c|c|c|c|c|c|c|c|}
\hline \multirow[t]{2}{*}{ CASE } & \multicolumn{4}{|c|}{ Convivial Network (CN) } & \multicolumn{4}{|c|}{ Friendship Network (FB) } & \multicolumn{2}{|c|}{ Heterogeneity } \\
\hline & Size & $\begin{array}{c}\text { Other } \\
\text { Nationals }\end{array}$ & $\begin{array}{l}\text { Notionsl } \\
\text { E-I imdex } \\
\text { (a) }\end{array}$ & $\begin{array}{l}\text { Ianguzge } \\
\text { E-I index } \\
\text { (b) }\end{array}$ & Size & $\begin{array}{c}\text { Other } \\
\text { Nationals }\end{array}$ & $\begin{array}{l}\text { National } \\
\text { E-I index } \\
\text { (c) }\end{array}$ & $\begin{array}{l}\text { Ianguage } \\
\text { E-I index } \\
\text { (d) }\end{array}$ & $\begin{array}{l}C n \\
\text { (e) }\end{array}$ & $\begin{array}{l}F n \\
\text { (f) }\end{array}$ \\
\hline $2[2 x+2$ & 17 & 14 & 0.65 & 0.05 & 7 & 6 & 0.71 & -0.14 & 0.464 & 0.245 \\
\hline Tyylz & 18 & 11 & 0.22 & -1 & 15 & 11 & 0.47 & -1 & 0.717 & 0.729 \\
\hline Andres & 33 & 15 & -0.09 & -0.21 & 8 & 5 & 0.25 & 0 & 0.642 & 0.718 \\
\hline Nocko & 23 & 14 & 0.22 & 0.04 & 13 & 8 & 0.23 & 0.23 & 0.608 & 0.674 \\
\hline Manibel & 24 & 14 & 0.17 & 0.17 & 13 & 8 & 0.23 & 0.23 & 0.531 & 0.556 \\
\hline Jesus & 34 & 21 & 0.24 & -0.24 & 20 & 12 & 0.20 & -0.50 & 0.636 & 0.685 \\
\hline $2 \log x$ & 42 & 25 & 0.19 & 0 & 39 & 22 & 0.13 & 0.08 & 0.559 & 0.570 \\
\hline Migual & 28 & 20 & 0.48 & 0 & 18 & 10 & 0.11 & -0.11 & 0.768 & 0.691 \\
\hline Adrian & 32 & 17 & 0.06 & -0.75 & 30 & 16 & 0.07 & -0.73 & 0.622 & 0.629 \\
\hline Crnmen & 36 & 19 & 0.06 & -0.61 & 17 & 9 & 0.06 & -1 & 0.701 & 0.671 \\
\hline Fenuy & 53 & 36 & 0.36 & -0.47 & 12 & 6 & 0 & -0.67 & 0.708 & 0.658 \\
\hline Lalo & 36 & 15 & -0.17 & -0.22 & 29 & 14 & -0.03 & -0.24 & 0.592 & 0.635 \\
\hline Mlean & 31 & 13.4 & 0.20 & -0.27 & 18.4 & 10.6 & 0.20 & -0.32 & 0.685 & 0.626 \\
\hline
\end{tabular}

4.10 For example Claire, 34 (Table 2 - stable), an English teacher in Madrid confessed that her level of Spanish was not great, which prevented her from having a wider convivial horizon 'that's my problem, yeah, working in English, English boyfriend and English speaking friends'. However, stable horizons tend to be older and their relationships with 'other nationals' tend to be more established, mirroring their more stable circumstances in terms of career, relational status and settlement.

4.11 The cases current social activity in Madrid or Manchester was decisive in the degree of heterophily and heterogeneity across the sample. Younger single participants tended to name more companions in general, and with it, depending on their social interests and occupations (working in multinational companies with diverse workforces), named more 'other nationals' as colleagues (Q3) and companions (Q2). While older more settled participants in stable relationships tended to name fewer companions, hence when named, 'other nationals' were more likely to be confidants (Q1). Additionally, they tended to be more established in terms of longevity, and as such were often more embedded within the network in terms of their degree centrality.

4.12 The biographical structuring of the network enabled participants to articulate elements of their socialization and the consequent changes in their sociability more fully. With a diverse sample in terms of age, mobility and settlement, relational status, etc. both different and similar effects of certain transitional events could be fully explored. For example, Laura, reflected on changes in her network and sociability in light of her move to Madrid with her partner the previous year, captured in the chapter title 'Into the Sunshine'. Laura outlined that she had recently been disappointed with her 'best friend' and confidant back in the UK who had not yet committed to visit her in Madrid.

...it made me think right, ok, screw you I'm going to put some more time and energy into the people here and give
them the, you know, (N. a chance) yeah, give them a chance really so that's what I've been doing, and it works, I
think it works if you just give people a little bit of vulnerability or you know something and they grab onto it (Laura 31)

4.13 However, this discussion also made her realise what she was currently missing in the city.

\begin{abstract}
So I think in terms of actually meeting people it's really easy, I don't know yet how easy it is to actually make proper friends, and I don't know how, I don't know how, how much I need to have proper friends, and also if I think all those people there, well they are people I've been friends with for years, $n$ years $n$ years and been through a load of shit with so that doesn't just come does it? (N. No) it does take time to have that kind level of closeness and for people to really know you as well, so that's what I'm missing here at the moment... (Laura 31)
\end{abstract}

4.14 The method enabled a more detailed interpretation of the link between socialization and sociability, with the narratives providing meaning behind the network measures. For instance, Laura's biographical network had gone from being completely homophilous (-1) in terms of nationality prior to Madrid, to -0.67 after a year in Madrid, with two-thirds of all new relations in her final chapter 'other nationals'.

4.15 Through building a case file on each person and tabulating aspects of the biography and the network, and then by comparing cases, clear trends and dynamics emerged. Further to nationality and language (horizons), the networks were compared in terms of social class, ethnicity, age, religion, sexual orientation (depths). The life histories (paths) were analysed independently and in relation to the networks. Each dimension 
added a further layer of analysis that thickened the description.

4.16 For instance, with cases outlining their reasoning for naming people with a different social class (Q4) and who they considered different to themselves (Q5), and by identifying alter on the visualization with different ethnicity etc. different paths through which horizons and depths emerged were identified. It was clear among many cases that horizons and depths correlated. By exploring these correlations, the significance of schooling, mobility, family, romances, foreign studies, etc. on the composition of networks were captured. Through comparing the consequences of similar and different life events among cases, their openness and willingness to engage with 'others' was uncovered and interpreted within a broader biographical and social context.

4.17 With the networks varying in size between cases, comparative structural analysis in terms of density and degree centrality was reduced. Instead, structural analysis was used to explore details within individual cases, such as the exploring the degree centrality of alter within the network and their centrality or role in the life history.

\section{Narrative Analysis and BNA}

4.18 While the life histories were continually cross-referenced when comparing the networks, they also stood as an independent data source for narrative analysis. Interviewees responded to the autobiographical prompts with considerable enthusiasm and imagination, producing informative chapter titles and rich narratives. Three significant and interconnected strands emerged from a comparative analysis of the narratives; national family culture, family structure; and sense of belonging during childhood and adolescence. These strands resulted in a range of possible paths that produced both similar and distinctive network compositions and structures. These strands had clearly impacted the biographical distribution of alter across the convivial and friendship networks, and alter-alter ties across life chapters and social contexts. The biographical structure of the network facilitated this co-analysis during the interview and further analysis after the interview.

4.19 With the number of chapter titles varying from 4 to 15 between cases, a systematic method to compare and present the biographical evolution of the networks was required. Through cross referencing and comparing cases, the richness of the narratives enabled the chapters and alter located within each to be allocated to one of five life stages; childhood (c), adolescence (a), further studies (s), working life 1 (w1), and working life 2 (w2). Hence the comparative framework was produced using a grounded methodology drawing on both quantitative and qualitative data. The biographical distribution of alter added a further contextual layer through which to analyse and interpret the cosmopolitan conviviality of cases.

4.20 For instance cases with a greater distribution of alter in childhood and adolescence (see Figure 6 - Case $A^{[3]}$ ) tended to narrate positive experiences in regards to these life stages, exemplified in chapter titles such as 'Avila a happy place' and 'The Good Life'. They also tended to identify their closest confidants and companions in these initial chapters and life stages. However, in contrast, their transition to university and adulthood had at times proved challenging, reflected in titles such as 'Time to Grow Up?', 'Life Gets Complicated' and 'The England Years Part 1: How to survive working part time and earning the minimum wage'. Their narratives tended to be more nostalgic, longing to replicate the settled familial foundations from which they had originated.

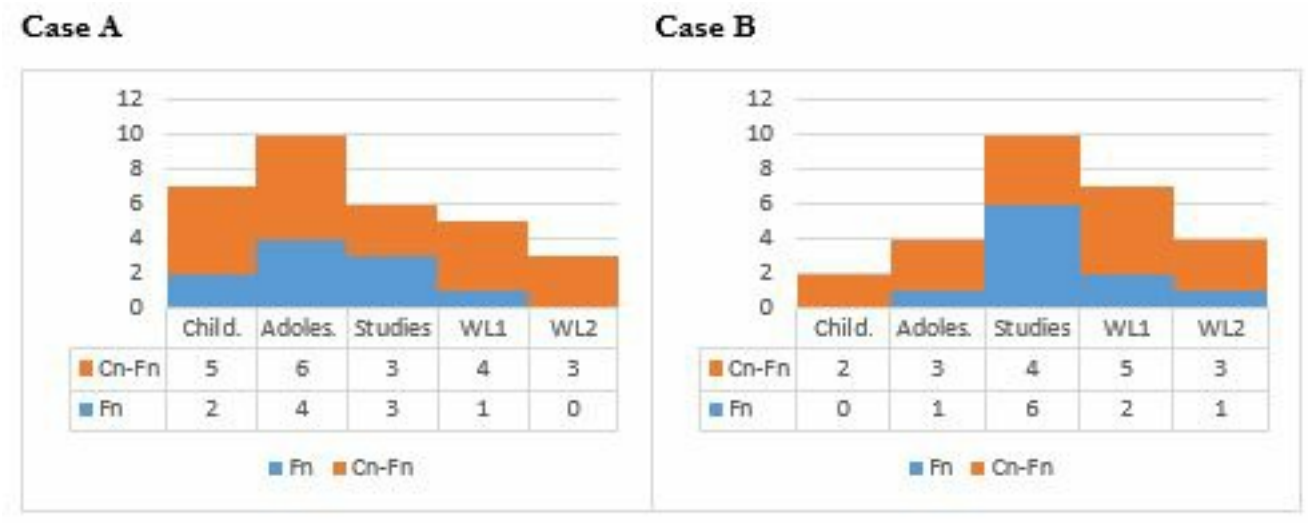

Figure 3. Biographical Distribution of Alter

4.21 Whereas cases that had a greater distribution of alter after adolescence (Case B), with a significant number of friends from university (s), at times articulated feelings of not always fitting in at school or their neighbourhood, or troublesome experiences during childhood and adolescence. This resulted in them often 
feeling 'the other'. Hence their life story narratives expressed the desire to escape their localities and broaden their horizons through further studies (s) and working life (w1\&2). Their experiences of not fitting in at school, and family break-ups were reflected in chapter titles such as 'Nobody said it was easy', 'That's Bullshit' and 'The Divorce of My Parents'.

4.22 Narrative differences between the Spanish and British were also evident in the biographical distribution of their networks, with the Spanish leaning to Case A and the British to Case B. While the British tended to narrate a clearer path from home to world (Tuan 1996), with themselves as the main dominant protagonist, the Spanish narratives while less lineal and cohesive, and paid greater attention to the significance of others on their life path. These differences stemmed from distinct national family cultures. A reoccurring theme among the Spanish women in Madrid and Manchester were their struggles in gaining independence and distance from the family, both emotionally and geographically. This constituted a significant theme in Carmen's (Table $3-$ wide) life history and chapter titles, such as 'Living in the city BUT under control'.

So I finished in three years, I finished because it's like my parents wanted that, I've done it, so it's like I got a degree, so with that it's something, and with that as well I can work and my parents are happy and 'my daughter is degree in labour law', so it's something, and then it's like, ok, I will do it, but now I realise like I not been myself in those three years, I'd not been myself, I always wanted to do things I got here (points to sociogram) but I could not find out (Carmen, 26)

4.23 In contrast to cases with more content childhoods and unified family structures (Case A), these Spanish women tended to identify stronger with confidants and companions in latter chapters in which they had gained further independence and broadened their horizons. Further qualitative structural analysis of the network visualisations, such as the extent to which alter-alter ties spanned across chapters, and social and national contexts, shed further light on people's paths and narratives. For example, in Mark's network (Table2 - stable), ties between alter in the UK and Madrid, where he had lived with his Spanish wife for five years, were weak and few. As such he experienced some dislocation between the two national contexts,

...the main thing is that from my point of view is that it feels like a different life... here, but the people that I remember most clearly were from when I was growing up. (Mark 30)

4.24 While patterns emerged between the life histories and the networks, in many instances contradictions and dissonance between the two appeared, which in turn generated further discussion during the interview and enriched the analysis. Through exploring symmetries and anomalies between the narrative strands and structure (paths) and the network composition and structure (horizons and depths), four broad but distinctive spheres of sociability emerged in relation to cases' openness and willingness to engage with 'others'.

\section{Presenting the Data}

4.25 The case of Laura, introduced earlier, is now more fully presented to outline the utility of the biographical network method for sociological analysis. Vennmaker (Kronenwett \& Schonhuth 2011) was used to visualise the networks for publication as it enables the use of concentric circles. Here they represent the five life stages; childhood (c), adolescence (a), further studies (s), working life 1 (w1), working life 2 (w2) that were generated for comparative biographical network analysis.

\section{Laura - Dislocation or Relocation}

4.26 Laura's six chapters are allocated accordingly to the five life stages in Figure 4 below. The autobiographical title 'People Lost and Found' was given at the end of the interview, which in large part reflects the changes in her sociability in light of her move 'Into the Sunshine' of Madrid. The relations generated from the life history are identified by a purple shadow, which in this case are all co-nationals (blue nodes). The biographical distribution of alter is even across the $\mathrm{Bn}, \mathrm{Cn}$ and $\mathrm{Fn}$, with alter named under Q1, Q2 and Q6 identified by nodes with a grey shadow. 


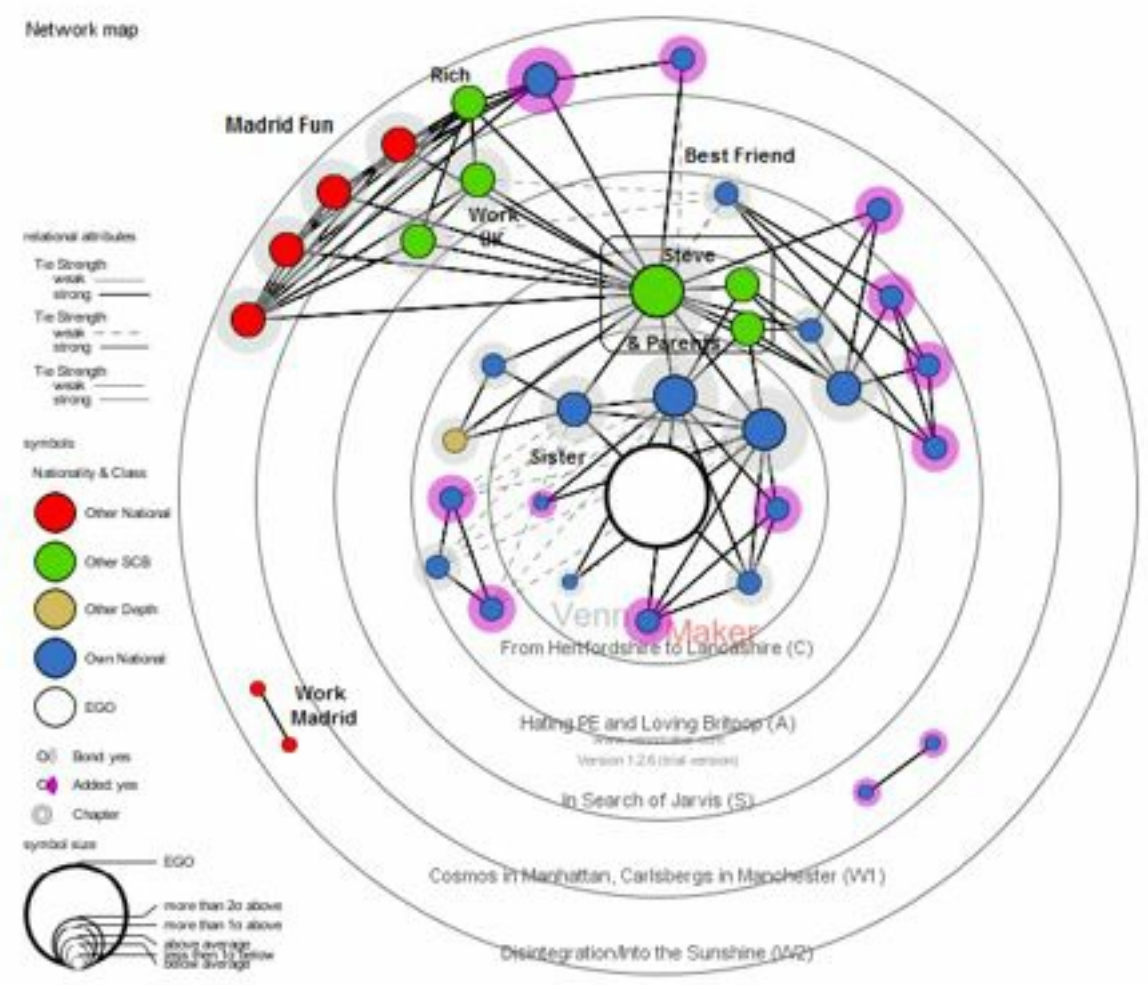

Figure 4. 'People Lost and Found'

4.27 As previously noted, the entrance of 'other nationals' coincides with her move to Madrid (red nodes) in the groups 'Work Madrid' and 'Madrid Fun' - the latter which she enjoys socialising and travelling with (Q2). Having the previous summer organised a trip travelling around southern Spain with two people from 'Madrid Fun', two UK work colleagues from 'Carlsberg in Manchester', and her partner Steve - the most central alter in the network (node size) ${ }^{[4]}$ - strong ties (full black lines) now bridge biographical chapters and geographical contexts in her network.

4.28 In terms of narrative structure, Laura outlined that the move from 'Hertfordshire to Lancashire' in childhood made her the new kid in school, and at times she struggled to fit in - 'Hating PE and loving Britpop'. As such only family members and friends of her family remain from childhood. However, through partaking in summer theatre camps during adolescence she was able to find people with similar interests, and she maintains good friends with people from this period. It was on one summer camp she first befriended Steve, her now partner of five-years.

4.29 Laura self-identified as middle-class, and named Steve and his parents, Rich, a fellow Brit in Madrid, and a former UK work colleague as working-class, and a further colleague as upper middle-class (green nodes). Interestingly, the only people she names with a different social-class background are 'co-nationals', and are based on a British cultural understanding of class. This pattern confers with findings across the sample, where lengthier settlement and engagement in the host culture is required to recognise class differentials among natives. For the Spanish in Manchester, this meant adopting a British social-class framework and naming predominantly British people on this prompt. The fact that I, a British researcher posed the prompt, was shown in some cases to have influenced their responses and was subsequently incorporated in the analysis. The Spanish in Madrid tended to name co-nationals with a higher social class due to either their family wealth or professional occupation and income, i.e. lawyers and doctors. These findings emerged due to the method's biographical structure and the participant-aided approach taken.

4.30 The depth in terms of social class within Laura's network arguably materialised during adolescence, a period from which she also identifies a close gay friend (yellow node = other ethnicity, religion, age etc.) Yet caution is required in interpreting findings based on attributes such as class and sexual-orientation, as they are experienced relationally (Bottero 2004, De Federico de la Rua 2007). Actors, both ego and alter, are moving targets, and as such attributes, or how 'self' identifies 'others' change with time (Maya-Jariego \& Armitage 2007). Hence, while Laura named people of a different social class and identified someone with a different sexual orientation, these were based on her current location and knowledge. In many cases, depths within networks 
came via leisure activities such as playing sport during adolescence or attending language exchange events (e.g. Rich in Laura's network), and not via their education or their workplaces.

4.31 Laura's love for Britpop led her 'In Search of Jarvis' Cocker during further studies (s) at university. While an active social period in her narrative that generated new alter to be included in the visualization, she only named her 'best friend' in this period via the name generator. Laura's disappointment regarding her 'best friend' not visiting is coupled to her peripheral network position. With only a few weak ties (faint dashed lines), her 'best friend' is not embedded within the network and as such Laura needs to dedicate more emotional energy to maintain this relationship. Laura's earlier quotes regarding her changing sociability reflect the context of both dislocation and new opportunities that arise through relocation.

\section{Concluding Discussion}

5.1 As illustrated by Laura, stories embedded within the networks take on greater meaning when placed in relation to their life history. The temporal framework enables greater clarity to analyse network dynamics, and the effects thereof. The tool opens new perspectives for sociological analysis, particularly the link between narrative structure and network composition and structure.

5.2 Through situating and conducting the life story interview prior to the visualization, the person in front of the network is allowed to first emerge. Taking this approach helped to develop rapport and a conversation with a purpose, whereby the visualization was used as an aid to further enlighten the analysis. In retrospect the survey tool should have followed the interview protocol, with the autobiographical prompt preceding the name generator so as not to potentially bias the chapter names. Further, the name generator should permit respondents to name confidants (Q1) also as companions (Q2) to improve the validity and reliability of the tool. While clarification of the protocol was often necessary during the interview, interviewees engaged positively and enjoyed the reflexivity that it encouraged. This enabled reliable data to be produced with minimal burden, and additional contextual data to be incorporated in the analysis. The approach taken and protocol is an efficient alternative to more rigorous contextual based name generator tools (Bidart and Charbonneau 2011) that may run the risk of qualitatively over burdening the interviewee.

5.3 The case of Laura demonstrates that by keeping the life story interview independent of the network, and the biographical structure, the data produced is both detailed and comprehensive, and as a result enables comparison. By encouraging the interviewee to reflect on and analyse their network in relation to their life history in the final part of the interview, further insights and layers of interpretation arise. This was essential for developing the five life-stage comparative framework, although some subjectivity of the chapters was lost. The iterative conversation between the data sets generates methodological reflexivity that opens new perspectives for sociological analysis.

5.4 The biographical network method was designed specifically to explore the evolution of 'self' in relation to their stance towards 'others'. The metaphor, model and protocol, like any network method, produced particular presentations of 'self' - or certain types of abstractions (Edwards 2010). And yet while the life history and the network are both retrospective accounts, they each help unpack the provisionality of the other, enabling more to be seen.

5.5 Similarly, the method is articulated from a position of retrospection, and suggests a coherent and lineal development of the biographical network method. While no life or process is that simple, by starting with the person and not the network, the method was able to expose repetition, contradictions and dissonance, and unpack the messiness and complexity of social life. The further temporal structuring and organisation of social networks is a warranted endeavour. The next chapter for the BN method is yet to unfold. However, the approach and innovations hereto, resulting in new analytical perspectives, suggest that a fruitful area for further exploration is the relationship between network evolution and narrative structure.

\section{Notes}

Where -1 denotes a completely homophilious network and +1 a completely heterophilious network. 
socio-cultural categories were created from the nationality attribute data; Co-Nationals (British/Spanish), European, Anglo-Saxon (North American, Australia, New Zealand), Hispanic (Central \& Latin America), African/Caribbean and Asian. By using 'co-nationals' as one category within the calculation, the measures of national homophily and socio-cultural heterogeneity are correlated.

Case A and B are aggregate examples of cases used to demonstrate the relationship between the biographical distribution of alter and narrative structure and characteristics

Calculated and determined using average node degree and standard deviation (Strong ties $=2$, Weak ties $=1$ ). This method was used in order to maintain comparability between different network sizes for purposes of presentation

\section{References}

BAGNOLI, A. (2009) Beyond the standard interview: the use of graphic elicitation and arts-based methods, Qualitative Research, 9(5), p. 547-570. [doi:10.1177/1468794109343625]

BARRERA, M. (1980) A method for the assessment of social support networks in community survey research. Connection, 3, p. 8-13.

BELLOTTI E. (2014). Qualitative Networks: Mixed methods in sociological research. Routledge.

BIDART, C. \& Charbonneau, J. (2011). "How to Generate Personal Networks: Issues and Tools for a Sociological Perspective", Field Methods, 23 (3), p. 266-286. [doi:10.1177/1525822X11408513]

BIDART, C. \& Lavenu, D. (2005) Evolutions of personal networks and life events,Social Networks, 27 (4), p. 359376. [doi:10.1016/j.socnet.2004.11.003]

BOISSEVAIN, J. (1979) Network Analysis: A Reappraisal, Current Anthropology, 20 (2), p. 392-394. [doi:10.1086/202277]

BORGATTI, S.P., Everett, M.G. and Freeman, L.C. (2002)Ucinet for Windows: Software for Social Network Analysis. Harvard, MA: Analytic Technologies.

BOTTERO, W. (2004) Class identities and the identity of class, Sociology, 38 (5), p. 985-1003. [doi:10.1177/0038038504047182]

BRANNEN, J. \& Nilsen, A. (2011) Comparative Biographies in Case-bases Cross-national Research: Methodological Considerations, Sociology, 45(4), p. 603-618. [doi:10.1177/0038038511406602]

BURGESS, R.G. (1984) In the Field: An Introduction to Field Research London: Allen and Unwin [doi:10.4324/9780203418161]

COOLEY, C.H. (1983) Human Nature and Social Order, New York: Schocken Books.

CROSSLEY, N. (2010) The Social World of the Network: Combining Quantitative and Qualitative Elements in Social Network Analysis, Sociologica, 1.

DE FEDERICO DE LA RUA, A. (2007) Networks and Identifications: A Relational Approach to Social Identities, International Sociology, 22 (6), p. 683-699. [doi:10.1177/0268580907082247]

DEGENNE, A. \& Lebeaux, M-O. (2005) The dynamics of personal networks at the time of entry into adult life, Social Networks, 27 (4), p. 337-358. [doi:10.1016/j.socnet.2004.11.002]

DOMINGUEZ, S. \& Hollstein, B. (2014)Mixed Methods Social Network Research: Design and Applications, Cambridge: Cambridge University Press. [doi:10.1017/CBO9781139227193]

EDWARDS, G. (2010) Mixed-Method Approaches to Social Network Analysis. Review Paper,ESRC National Centre for Research Methods. 
EMMEL, N. (2008) Participatory mapping: an innovative sociological method, Real Life Methods Toolkit. http://www.reallifemethods.ac.uk/publications/toolkits/2008-07-toolkitparticipatory-map.pdf.

GROSSETTI, M. (2005) Where do social relations come from? A study of personal networks in the Toulouse area of France, Social Networks, 27(4), p. 289-300. [doi:10.1016/j.socnet.2004.11.004]

HANNERZ, U. (1996) Transnational Connections: Culture, People, Places. London: Routledge.

HENDERSON, S., Holland, J., McGrellis, S., Sharpe, S. \& Thomson, R. (2007)Inventing Adulthoods: a biographical approach to youth transitions, London: Sage.

HOGAN, B., Carrasco, J.A. \& Wellman, B. (2007) Visualizing Personal Networks: Working with Participant-Aided Sociograms, Field Methods, 19(2) p. 116-144. [doi:10.1177/1525822X06298589]

HOLLAND, J. and Thomson, R. (2009) Gaining a perspective on choice and fate: revisiting critical moments, European Societies, 11(3), p. 451-469. [doi:10.1080/14616690902764799]

LAUMANN, Edward O 1973. Bonds of Pluralism: The Form and Substance of Urban Social Networks, New York: John Wiley and Sons.

MASON, J. (2002) Qualitative Researching, London: Sage.

MASON, J. (2006) Mixing methods in a qualitatively driven way, Qualitative Research, 6(1), p. 9-25. [doi:10.1177/1468794106058866]

MAYA-JARIEGO, I. \& Armitage, N. (2007) Multiple Senses of Community in Migration and Commuting: The Interplay between Time, Space and Relations International Sociology 22(6), p. 743-766.

MEAD, G.H. (1984) George Herbert Mead on Social Psychology, London: University of Chicago Press.

MOODY, J.W. \& Healy, K. (2014) Data Visualisation in Sociology,Annual Review of Sociology, 40.

RYAN L., Mulholland J., Agoston A. (2014). Talking ties: Reflecting on network visualisation and qualitative interviewing. Sociological Research Online, 19 (2) 16 http://www.socresonline.org.uk/19/2/16.html 10.5153/sro.3404. [doi:10.5153/sro.3404]

TUAN, Y (1996) Cosmos and Hearth: A Cosmopolite's Viewpoint University of Minnesota Press. 